\title{
Phase Diagram of a Two-Species Lattice Model with a Linear Instability
}

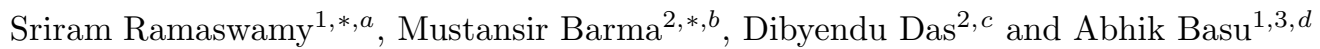 \\ ${ }^{1}$ Centre for Condensed Matter Theory, Department of Physics, Indian Institute of Science, Bangalore 560012, India, \\ ${ }^{2}$ Department of Theoretical Physics, Tata Institute of Fundamental Research, Homi Bhabha Road, Mumbai 400005, India, \\ ${ }^{3}$ Poornaprajna Institute of Scientific Research, Bangalore, India
}

\begin{abstract}
We discuss the properties of a one-dimensional lattice model of a driven system with two species of particles in which the mobility of one species depends on the density of the other. This model was introduced by Lahiri and Ramaswamy in the context of sedimenting colloidal crystals, and its continuum version was shown to exhibit an instability arising from linear gradient couplings. In this paper we review recent progress in understanding the full phase diagram of the model. There are three phases. In the first, the steady state can be determined exactly along a representative locus using the condition of detailed balance. The system shows phase separation of an exceptionally robust sort, termed strong phase separation, which survives at all temperature. The second phase arises in the threshold case where the first species evolves independently of the second, but the fluctuations of the first influence the evolution of the second, as in the passive scalar problem. The second species then shows phase separation of a delicate sort, in which long range order coexists with fluctuations which do not damp down in the large-size limit. This fluctuation-dominated phase ordering is associated with power law decays in cluster size distributions and a breakdown of the Porod law. The third phase is one with a uniform overall density, and along a representative locus the steady state is shown to have product measure form. Density fluctuations are transported by two kinematic waves, each involving both species and coupled at the nonlinear level. Their dissipation properties are governed by the symmetries of these couplings, which depend on the overall densities. In the most interesting case, the dissipation of the two modes is characterized by different critical exponents, despite the nonlinear coupling.
\end{abstract}

\section{INTRODUCTION}

Much recent progress in the general area of nonequilibrium steady states has come about by focusing on simple driven lattice-gas models which have the same symmetries as the more complex physical situations they represent. For instance, the asymmetric exclusion process - a simple model of hard-core particles performing biased random walks - is representative of a class of current-carrying states and growth processes involving a single scalar field [1]. Now, there are several examples of physical processes which involve two coupled scalar fields [2] 7]; for such situations, the corresponding lattice model involves two sets of Ising variables, with the time evolution of each species depending on the instantaneous configuration of the other. Such systems can display interesting, new types of steady states, and in this paper we discuss their occurrence within a recently introduced lattice model of coupled fields.

This model was initially proposed by Lahiri and Ramaswamy [7] (hence referred to as the LR model) in connection with collective effects in sedimentation, the process by which heavier particles settle in a lighter fluid. Interactions between sedimenting particles are mediated by the fluid [8 10], and give rise to a coupled dynamics of two fields, namely the concentration (the particle number density) and the tilt field (the orientation of the principal axes of the particle distribution). A distinctive aspect of the inter-field coupling in this case is that within a hydrodynamic description, the coupling involves linear terms, in addition to more customary nonlinear coupling terms. This essential aspect is captured by the 1-d LR lattice model. Linear couplings can also arise in other contexts, for instance in reduced models of magnetohydrodynamics [11], drifting flux lattices in superconductors [12], and the motion of stuck and flowing grains on a sandpile [13]. The essential aspect of linear coupling is captured by the 1-d LR lattice model. In the sedimentation problem, analysis of the linearized hydrodynamic equations points to an instability in a certain regime, but leaves open the question of the ultimate steady state reached by the system. The lattice model allows this question to be answered; the instability can be studied by varying the transition rates within the model, and the resulting steady state can be analysed and characterized [7,14].

The instability in question is towards large-scale clustering, and the question arises to what extent the phenomenon resembles phase separation, familiar from equilibrium statistical mechanics. In fact, the nature of phase separation in nonequilibrium steady states is an area of great interest at present [15]17], and the study of the LR model proves quite illuminating in this context. The unstable phase turns out to be strongly phase separated, much as in the three-component model of Evans et al [18,19]. The phase separation is robust, and survives at all temperatures. The behaviour is quite different, however, at the threshold of the instability, which corresponds to the particularly simple situation in which one of the two fields evolves autonomously while the other is driven by the fluctuations 
of the first, as in the passive scalar problem. There is phase ordering in this case as well, but it is quite delicate. Fluctuations of the order parameter are very strong, but do not destroy the ordered phase [20]; rather, they change its character in an essential way, leading to the breakdown of familiar characteristics such as the Porod law for the decay of scaled correlations. Finally, moving away from the threshold, even the disordered regime of the model has some surprises to offer. In this regime, the density is homogeneous on a macroscopic scale, but fluctuations are transported ballistically by two sets of kinematic waves [21]. The waves are coupled nonlinearly, yet under special symmetry-determined conditions (i.e., on a special locus in parameter space) the dissipation of each is governed by a distinct critical exponent [23]. This is the first such instance to come to light in a system with nonvanishing nonlinear coupling. In the kinematic wave phase in general, the equations are a one-dimensional reduced model for the dynamics of small fluctuations of a flux lattice drifting through a type II superconductor [12], and also serve as a generalised Burgers equation for magnetohydrodynamics (MHD) [1].

In the remainder of this section, we discuss the occurrence of the instability within a linearized hydrodynamic description, and then define the LR lattice model and briefly discuss its phase diagram. In the sections that follow, we discuss respectively strong phase separation (in the unstable regime), fluctuation-dominated phase separation (at the threshold), and kinematic waves and their decay (in the stable regime).

\section{A. Hydrodynamic Description and Instabilities}

The hydrodynamic equations of a coupled-field system involve the density fluctuations $\delta \rho_{1,2}(x, t) \equiv \rho_{1,2}(x, t)-\rho_{1,2}^{o}$ of the two species in question. When dealing with conserved fields, the equations governing their time evolutions may be derived from the continuity equations $\partial \rho_{1} / \partial t+\partial J_{1} / \partial x=0$ and $\partial \rho_{2} / \partial t+\partial J_{2} / \partial x=0$, where $J_{1}\left(\delta \rho_{1}, \delta \rho_{2}\right)$ and $J_{2}\left(\delta \rho_{1}, \delta \rho_{2}\right)$ are the currents of particles of species 1 and 2 respectively. We may proceed by writing each of the currents $J_{1}$ and $J_{2}$ in terms of a systematic part $J_{1,2}^{s y s}$, a diffusive part $-D \partial \rho_{1,2}(x, t) / \partial x$, and noise $\eta_{1,2}$. The coupling between the two species arises from the systematic parts, for each of $J_{1}^{s y s}$ and $J_{2}^{\text {sys }}$ depend both on $\delta \rho_{1}$ and $\delta \rho_{2}$. We obtain the requisite equations on expanding in powers of the density fluctuations. We find it convenient to write these equations in terms of the integrated density fields $h_{1,2}(x, t)=\int^{x} \delta \rho\left(x^{\prime}, t\right) d x^{\prime}$. Then, to second order, we obtain

$$
\begin{aligned}
& \frac{\partial h_{1}}{\partial t}=c_{11} \frac{\partial h_{1}}{\partial x}+c_{12} \frac{\partial h_{2}}{\partial x}+D_{1} \frac{\partial^{2} h_{1}}{\partial x^{2}}+\lambda_{1}\left(\frac{\partial h_{1}}{\partial x}\right)^{2}+\mu_{1}\left(\frac{\partial h_{2}}{\partial x}\right)^{2}+\nu_{1}\left(\frac{\partial h_{1}}{\partial x}\right)\left(\frac{\partial h_{2}}{\partial x}\right)+\eta_{1}(x, t) \\
& \frac{\partial h_{2}}{\partial t}=c_{21} \frac{\partial h_{1}}{\partial x}+c_{22} \frac{\partial h_{2}}{\partial x}+D_{2} \frac{\partial^{2} h_{2}}{\partial x^{2}}+\lambda_{2}\left(\frac{\partial h_{1}}{\partial x}\right)^{2}+\mu_{2}\left(\frac{\partial h_{2}}{\partial x}\right)^{2}+\nu_{2}\left(\frac{\partial h_{1}}{\partial x}\right)\left(\frac{\partial h_{2}}{\partial x}\right)+\eta_{2}(x, t) .
\end{aligned}
$$

Let us first examine the effect of keeping only the linear first derivative terms on the right hand side of the above equations. These terms, which are of primary interest to us here, were absent on symmetry grounds in coupled-field problems involving directed polymer motion and surface growth, considered respectively in references [2, 何, and [5.60.

Let the eigenvectors of the $2 \times 2$ matrix $\left(\begin{array}{ll}c_{11} & c_{12} \\ c_{21} & c_{22}\end{array}\right)$ be $e_{+}$and $e_{-}$, and let the corresponding eigenvalues be $c_{+}$and $c_{-}$. There are three cases to consider:

(A) Stable: If $c_{+}$and $c_{-}$are real (i.e. if $\left.\Delta \equiv\left(c_{11}-c_{22}\right)^{2}+4 c_{12} c_{21}>0\right)$, they represent the speeds of two waves. The two waves involve the eigenvectors $e_{+}$and $e_{-}$, each of which is composed of a linear combination of $h_{1}$ and $h_{2}$. To proceed, rewrite Eqs. 11 in terms of $e_{+}(x, t)$ and $e_{-}(x, t)$. Notice that in the rest frame of each wave, the other wave moves with a finite speed $\left|c_{+}-c_{-}\right|$; it is impossible to make both stationary by a Galilean shift. The nonlinear terms describe the couplings between the two waves which govern their dissipation.

(B) Threshold: The discriminant $\Delta$ vanishes if one of the off-diagonal terms, say $c_{12}$, is zero. At the linear level, this would imply that the field $h_{1}$ evolves autonomously, but (since $\left.c_{21} \neq 0\right)$ the time evolution of the 'passive scalar' $h_{2}$ is influenced by $h_{1}$. There is a tendency for particles of type 2 to be driven together. In the corresponding lattice model, this system shows phase separation accompanied, however, by large-scale fluctuations.

(C) Unstable: There is an instability if $c_{+}$and $c_{-}$pick up an imaginary part (i.e. if $\Delta<0$ ). The solutions of the linear equations then have an unbounded exponential growth of fluctuations. This instability signals the advent of a new state which is qualitatively different from the statistically homogeneous state assumed at the outset. By studying the corresponding lattice model in the unstable regime, we find that the system undergoes macroscopic phase separation of a robust sort. 


\section{B. Lattice Model and Phase Diagram}

The Lahiri Ramaswamy model [7] is the lattice counterpart of the continuum equations discussed above, and analysing its phases helps us understand the behaviour of the system in each of the cases (A), (B) and (C) above.

The LR model is defined in terms of two sets of variables $\left\{\sigma_{i}\right\}$ and $\left\{\tau_{i-\frac{1}{2}}\right\}$ which reside on two interpenetrating sublattices; the former occupy the integer sites and the latter the half-integer bond locations of a one-dimensional lattice with $L$ sites. Each $\sigma_{i}$ and $\tau_{i-\frac{1}{2}}$ is an Ising variable taking on values \pm 1 . They represent discrete versions of the density and tilt fields in the sedimentation problem: If $\sigma_{i}$ is 1 , there is a particle $(+)$ at site $i$, and if $\sigma_{i}=-1$, there is no particle $(-)$. The variable $\tau_{i-\frac{1}{2}}=1$ and -1 , implies two values $/$ and $\backslash$ of the local tilt respectively. A typical configuration of the full system is thus: $+1-1-1+1-1+1+1+1-$.

Both sets of variables are conserved, i.e. $\sum \sigma_{i}$ and $\sum \tau_{i-\frac{1}{2}}$ and the associated densities $\rho_{1}^{o}=\sum\left(1+\sigma_{i}\right) / 2 L$ and $\rho_{2}^{o}=\sum\left(1+\tau_{i-\frac{1}{2}}\right) / 2 L$ are constant. The linear terms in the hydrodynamic equations lead us to consider a $\tau$-dependent local field which guides the $\sigma$-current and vice versa. Thus, for instance, the Kawasaki exchange dynamics of the adjacent spins $\sigma_{i}$ and $\sigma_{i+1}$ occurs at a rate which depends on $\tau_{i+\frac{1}{2}}$. The moves and the corresponding rates are depicted below:

$$
\begin{array}{lllll}
{[1]} & +\backslash- & \rightarrow & -\backslash+ & r_{1} \\
{[2]} & -\backslash+ & \rightarrow & +\backslash- & r_{2} \\
{[3]} & -/+ & \rightarrow & +/- & r_{1} \\
{[4]} & +/- & \rightarrow & -/+ & r_{2} \\
{[5]} & /-\backslash & \rightarrow & \backslash-/ & p_{2} \\
{[6]} & \backslash-/ & \rightarrow & /-\backslash & p_{1} \\
{[7]} & \backslash+/ & \rightarrow & /+\backslash & p_{2} \\
{[8]} & /+\backslash & \rightarrow & \backslash+/ & p_{1}
\end{array}
$$

The rates may be written succinctly as

$$
\begin{gathered}
W\left(\sigma_{i} \leftrightarrow \sigma_{i+1} ; \tau_{i+\frac{1}{2}}\right)=\frac{\left(r_{1}+r_{2}\right)}{2}-\frac{\left(r_{1}-r_{2}\right)}{4} \tau_{i+\frac{1}{2}}\left(\sigma_{i}-\sigma_{i+1}\right) \\
W\left(\tau_{i-\frac{1}{2}} \leftrightarrow \tau_{i+\frac{1}{2}} ; \sigma_{i}\right)=\frac{\left(p_{1}+p_{2}\right)}{2}+\frac{\left(p_{1}-p_{2}\right)}{4} \sigma_{i}\left(\tau_{i-\frac{1}{2}}-\tau_{i+\frac{1}{2}}\right) .
\end{gathered}
$$

In a simple correspondence to a hydrodynamic model, discussed in section IV below, the coefficients in the continuum description (Eq. 1) depend on the rates $p_{1}, p_{2}, r_{1}, r_{2}$, as also on the overall densities $\rho_{1}^{o}$ and $\rho_{2}^{o}$ of the two species. This correspondence allows us to demarcate each of the regimes (A), (B) and (C) discussed above in terms of the parameters of the lattice model.

Throughout the rest of the paper, we will assume that $r_{1}>r_{2}$. Thus particles tend to move preferentially downhill, and holes uphill. Holding the ratio $r_{1} / r_{2}$ fixed, it is then interesting to ask for the effect of varying $p_{1} / p_{2}$. The result is summarized in the phase diagram of Fig. 1, for the half-filled case $\sum \sigma_{i}=\sum \tau_{i}=0$. We discuss the characteristics of each phase in brief.

$p_{1}>p_{2}$ (the light grey region in Fig. 1): In the corresponding continuum equation, the diagonal coefficients $c_{11}$ and $c_{22}$ vanish, while $c_{12}$ and $c_{21}$ both have the same sign. Thus $\Delta$ is negative, corresponding to the unstable case (C). Since $p_{1}>p_{2}$, a local peak $(\bigwedge)$ tends to become a valley $(\backslash /)$ if a particle resides on it, while a valley tends to convert into a hill if a hole sits on it. These moves act in concert with the motion of particles down slopes (determined by $r_{1}>r_{2}$ ), to promote segregation of both kinds of spins. In fact, using the condition of detailed balance, the steady state can be determined exactly along the the line $r_{1} / r_{2}=p_{1} / p_{2}$ shown in Fig. 1 [14. The state shows phase segregation of a particularly strong sort, as discussed in section 2 .

$p_{1}=p_{2}$ (the thick central line in Fig. 1): This is the threshold case, with $c_{12}=0$ leading to vanishing $\Delta$. It describes a semi-autonomous problem in which the $\tau$ 's evolve independently of the $\sigma$ 's; they undergo a symmetric exclusion process. The $\sigma$ 's on the other hand obey the dynamics of Eq. 3. The system shows a particularly delicate sort of phase separation, which is accompanied by large-scale fluctuations in the steady state [20.22]. 


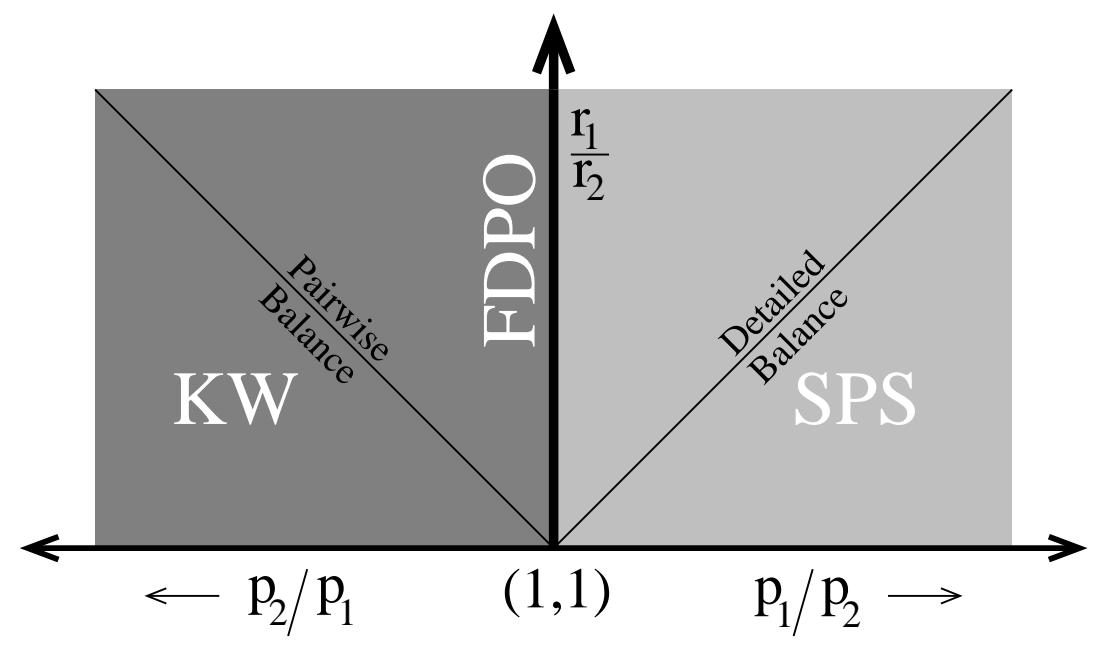

Figure 1:The phase diagram of the LR model at half-filling $\left(\sum \sigma_{i}=\sum \tau_{i-1 / 2}=0\right)$. The strongly phase separated phase (SPS) is separated from the kinematic wave phase $(\mathrm{KW})$ by the threshold, semi-autonomous line $p_{1}=p_{2}$ where there is fluctuation-dominated phase ordering (FDPO). The steady state can be found exactly along the line $r_{1} / r_{2}=p_{1} / p_{2}$ (using the condition of detailed balance) and along $r_{1} / r_{2}=p_{2} / p_{1}$ (using the condition of pairwise balance).

$\underline{p_{1}<p_{2}}$ (the dark grey region in Fig. 1): In this case $c_{12}$ and $c_{21}$ have opposite signs, so that $\Delta$ is positive. The tendency of particles to slide downhill is countered by the tendency of particle-rich valleys to become hills. The resulting steady state has a uniform macroscopic density and slope, while density fluctuations are carried by two kinematic waves, each corresponding to a mode which involves both $\sigma$ 's and $\tau$ 's. The steady state can be found exactly along the line $r_{1} / r_{2}=p_{2} / p_{1}$ shown in Fig. 1, by using the condition of pairwise balance [24]. Interestingly, the symmetries of the mode evolution equations can be tuned by varying the overall densities, and it is thereby possible to achieve a situation in which the dissipation of the two modes have different dynamical exponents in spite of being coupled at the nonlinear level [23].

\section{STRONG PHASE SEPARATION}

In the unstable phase of the LR model $\left(p_{1}>p_{2} ; r_{1}>r_{2}\right.$, the region marked SPS in Fig. 1), there is a bootstrap effect at work: the tendency of $\sigma$ particles to fall downhill into valleys works in tandem with the tendency of particlerich regions of the $\tau$ landscape to become valleys themselves. This results in the formation of ever deeper valleys and large-scale clustering of particles in them, leading ultimately to a phase separated state.

A similar sort of phase separation has been shown to occur in a three species ABC model in which a cyclic rule determines preferential rates of exchanges of adjacent particles [18,19. For the case with an equal number of A, B and $\mathrm{C}$ particles, the steady state was found exactly, using the condition of detailed balance.

In the LR model too, the exact steady state can be found [14] through a detailed balance argument in the case $\sum \sigma_{i}=\sum \tau_{i}=0, r_{1} / r_{2}=p_{1} / p_{2}$ (Fig. 1). This happens because although the problem is defined in terms of transition rates, it is possible to construct a Hamiltonian with respect to which detailed balance is valid. This Hamiltonian has the form

$$
\mathcal{H}=\epsilon \sum_{k=1}^{N} h_{k}\{\tau\} \sigma_{k}
$$

where $h_{k}\{\tau\}$ is a height field (the discrete analog of $h(x)$ in Eq. 11) defined by

$$
h_{k}\{\tau\}=\sum_{j=1}^{k} \tau_{j-1 / 2} .
$$

The steady state is thus described by an equilibrium Boltzmann measure, and a configuration $\{\sigma, \tau\}$ has weight $\exp (-\mathcal{H}\{\sigma, \tau\} / T)$ where $T$ is the temperature. This can be seen by noting that the energy change on interchanging neighbouring $\sigma$ spins is 


$$
\Delta E_{\sigma} \equiv \Delta E\left(\sigma_{i} \leftrightarrow \sigma_{i+1}\right)=\epsilon \tau_{i+\frac{1}{2}}\left(\sigma_{i}-\sigma_{i+1}\right),
$$

while the change of energy under exchange of $\tau$ spins is given by a similar expression with $\sigma$ 's and $\tau$ 's interchanged. Thus the ratio of Boltzmann weights of the configurations after and before an interchange of spins, say $\sigma_{i} \leftrightarrow \sigma_{i+1}$, is $\exp \left(-2 \Delta E_{\sigma} / T\right)$. This equals the ratio of forward to backward transition rates if the ratio $\epsilon / T$ is related to the rates $p_{1}$ and $p_{2}$ by

$$
\frac{p_{2}}{p_{1}}=e^{-2 \epsilon / T}
$$

The ratio $r_{2} / r_{1}$ of forward and backward transition rates involving the interchange of adjacent $\tau$ 's is also related to the same weight factor. Thus, provided $r_{1} / r_{2}=p_{1} / p_{2}$ holds, the steady state weight of a configuration is proportional to $\exp (-\mathcal{H}) / T$. Notice that $\mathcal{H}$ involves long-ranged couplings between spins $\sigma_{i}$ and $\tau_{j-1 / 2}$.

The ground state exhibits complete phase separation, corresponding to all $\sigma=1$ particles being at the bottom of the potential well formed by phase-separated $\tau$ spins. Pictorially, the state is

$$
-\backslash-\backslash-\backslash-\backslash+\backslash+\backslash+\backslash+1+1+1+1-1-1-1
$$

There are two interfaces across which there is a change of $\sigma$ spin values, and two more involving the $\tau$ 's. The spacing between the four interfaces is $L / 4$, and each is completely sharp in the limit $T \rightarrow 0$. The effect of nonzero temperature is to smear out each of the four interfaces over a finite length. The interfacial profile can be calculated [19], but for our purpose here it suffices to note that the interface width is of the order of $T$, and has basically no effect on the bulk of the spins. Thus in an infinite sample, phase separation survives at all $T$. This unusual behaviour can be traced to the fact that the energy grows super-extensively $\left(\sim(\text { length })^{2}\right)$, and thus dominates over the entropy at all temperatures. This allows the strictures against phase separation in equilibrium one-dimensional systems [25] to be avoided.

When the filling is changed away from $\sum_{i} \sigma_{i}=\sum_{i} \tau_{i}=0$, the condition of detailed balance no longer holds, and there is no description in terms of an effective Hamiltonian. Nevertheless, kinetic arguments can be given to show that phase separation survives even when the filling is changed. The extreme robustness of the phenomenon leads us to call it Strong Phase Separation.

The approach to the steady state is exceptionally slow. It occurs through a coarsening process which involves thermal activation over large barriers, so we expect a coarsening length growing logarithmically in time. Logarithmically slow coarsening was found in the ABC model as well [18].

In concluding this section, we mention a related model which has quite different behaviour. Recall that in the LR model, the mobility of each spin species depends on the local density of the other species. What if the mobility depends not on density, but a higher derivative of the density? A case in which the coupling is to the second derivative of the density can be analysed through detailed balance considerations, but this time, the Hamiltonian has shortranged interactions [26]. There is thus no phase ordering in this model at nonzero $T$, in contrast to the strong phase separation exhibited by the LR model in the unstable regime.

\section{FLUCTUATION-DOMINATED PHASE ORDERING}

In the marginal case $p_{1}=p_{2}, \quad r_{1}>r_{2}$ (the line FDPO in Fig. 1), the evolution of the $\tau$ 's proceeds independently of the $\sigma$ 's. The fluctuating $\tau$ landscape provides a source of nonequilibrium noise on the $\sigma$ 's which tend to fall downwards along local slopes and cluster in the valleys. Since the strong landscape fluctuations never cease, the clustering is never as complete as in the case of strong phase separation. Rather, as we will see below, the character of long-range order is strongly modified by fluctuations, hence the appellation Fluctuation-dominated Phase Ordering.

Starting from an initial random arrangement of the $\sigma$ particles, the extent of clustering can be estimated quite simply [20]: In any fluctuating surface characterized by a dynamical exponent $z$, there are rearrangements of the profile over length scales $\sim t^{1 / z}$ in time $t$. This sets the scale for the base lengths of new valleys which form in time $t$. In the case at hand, where the scaling properties of the surface are those of the Edwards-Wilkinson model [27], we have $z=2$. This valley base length should set the scale for spatial clustering of particles, so we expect the equal time correlation function to follow the scaling form

$$
\left\langle\sigma_{o}(t) \sigma_{o+r}(t)\right\rangle=C(r / \mathcal{L}(t)) \quad \text { with } \quad \mathcal{L} \sim t^{1 / z} .
$$

This is confirmed by numerical studies. This sort of scaling behaviour is a general characteristic of phase ordering dynamics, which describes the evolution from an initially disordered state to one which is phase separated [28]. 
However, the scaling function here is quite different from that in usual cases. Normally, with a conserved order parameter, $C(y)$ drops linearly for small $y \equiv r / \mathcal{L}(t)$, i.e. $C(y) \approx C_{o}\left(1-c_{1}|y|\right)$ as $y \rightarrow 0$. By contrast, we find $C$ shows a cusp for small values of $|y|$, i.e.

$$
C(y) \approx C_{o}\left(1-c_{1}|y|^{\alpha}\right)
$$

with $\alpha \simeq 0.5$ [20]. This is significant, since a linear dependence on $|y|$ (which goes under the name of the Porod law) is a simple consequence of having phases separated by sharp interfaces [28]. The breakdown of this law is the first pointer to the unusual character of the state that is developing.

In steady state, $C$ again shows a similar scaling form, with the coarsening scale $\mathcal{L}(t)$ being replaced by the system size $L$. The cusp for small argument remains unaltered. Turning to one-point correlation functions, a suitable measure 29] for our conserved spin system is the magnitude of the Fourier components of the density profile

$$
Q(k)=\left|\frac{1}{L} \sum_{j=1}^{L} e^{i k j} n_{j}\right|, \quad k=\frac{2 \pi m}{L} .
$$

where $n_{j}=\left(1+\sigma_{j}\right) / 2$ and $m$ runs over $1, \ldots, L-1$. A signature of an ordered state is that in the thermodynamic limit, the average values $\langle Q(k)\rangle$ approach zero for all nonzero $k$, but $Q(k \rightarrow 0)$ remains finite. We numerically monitored these averages $\langle\cdots\rangle$ over the ensemble of steady state configurations and found that $\langle Q(k)\rangle \rightarrow 0$ in the thermodynamic limit for fixed $k \neq 0$, but $\left\langle Q^{*}\right\rangle$ approaches a nonzero value, where $Q^{*}=Q\left(k=\frac{2 \pi}{L}\right)$.

$\left\langle Q^{*}\right\rangle$ provides a quantitative measure of phase separation. We find that $\left\langle Q^{*}\right\rangle \simeq 0.18$ indicating that the steady state is ordered, though not perfectly (a perfectly ordered state has $Q^{*} \simeq 0.32$ ). As a function of time, $Q^{*}$ fluctuates strongly, reflected in the fact that the distribution $P\left(Q^{*}\right)$ remains broad, with a root-mean-squared deviation that remains finite even as $L \rightarrow \infty$. One may ask whether phase ordering disappears when, in the course of its fluctuations, the value of $Q^{*}$ falls close to zero. The answer is in the negative. We observe that a dip in $Q^{*}$ is accompanied by a simultaneous rise in the value of either $Q(m=2)$ or $Q(m=3)$. This implies that whenever the system loses a single large cluster (making $Q^{*}$ small) either two or three such clusters appear in its place (making the values of $Q(m)$ for some small $m$ go up). Thus the system remains far from the disordered state, and always has a few large particle clusters which are of macroscopic size $\sim L$. A numerical study shows that the average size of the largest particle cluster $\simeq 0.14 L$.

We observe further that the particle and hole cluster size distributions in the steady state decay as a power-law:

$$
\operatorname{Prob}(l) \sim l^{-\theta}
$$

with $\theta \simeq 1$.8. This allows us to understand some features of the correlation functions discussed above. Within the independent interval approximation [30] which ignores correlations in the lengths of successive clusters, the two-point correlation function can be calculated and shown to have the scaling form of Eq. 8 [20]. The cusp in the scaling function is reproduced as well, with the cusp exponent $\alpha$ being related to $\theta$ by $\alpha+\theta=2$ within this approximation. On a qualitative note, the power law cluster distribution makes the interfaces between phases broad and structured, so that the argument which leads to the Porod law does not hold any longer.

The phenomenon of fluctuation-dominated phase ordering, with the characteristics of broad distributions of order parameter fluctuations, power-law cluster distributions, and cusps in the scaled two-point correlation functions, can be shown analytically to hold for a related simple model of surface depth fluctuations. Both steady state properties and time-dependent coarsening correlation functions can be calculated within this model [20,22], and the results bear out the picture of FDPO discussed above.

\section{THE KINEMATIC WAVE PHASE}

In the stable phase of the LR model $\left(r_{1}>r_{2}, \quad p_{2}>p_{1}\right)$, particle motion and landscape fluctuations work oppositely, so that the resulting state is disordered. The density of both $\sigma$ 's and $\tau$ 's is uniform, but the dynamics of density fluctuations is interesting, and this is the aspect we focus on.

To begin with, we note that the steady state can be shown exactly to have a product measure form, provided that $r_{1}=p_{2}$ and $r_{2}=p_{1}$ (the line marked Pairwise Balance in Fig. 1). To do this, choose new symbols to denote the values of $\sigma$ and $\tau$ : Use 1 if a site or bond is occupied by a + or $/$ and 0 for - or $\backslash$. Then the elementary moves reduce to $100 \rightarrow 001$ and $011 \rightarrow 110$ (with rate $r_{1}$ ), and the reverse moves $001 \rightarrow 100$ and $110 \rightarrow 011$ (with rate $r_{2}$ ). Now, in steady state the total probability flux into each configuration $C$ is equal to the total flux out of $C$. A sufficient condition for this is the condition of pairwise balance [24]: in-fluxes and out-fluxes must balance in pairs, 
i.e. for every out-flux $C \rightarrow C^{\prime}$, there is a unique in-flux $C^{\prime \prime} \rightarrow C$ such that $W\left(C^{\prime \prime} \rightarrow C\right) P_{s s}\left(C^{\prime \prime}\right)=W\left(C \rightarrow C^{\prime}\right) P_{s s}(C)$ where $W$ 's are transition rates, and $P_{s s}$ 's are steady state probabilities. Using intuition gained from particle hopping models, this condition can be shown to be valid [23] if $P_{s s}(C)=P_{s s}\left(C^{\prime \prime}\right)$. Thus every allowed configuration is equally likely in steady state, which implies that $P(C)$ is the product of independent site occupation probabilities in the thermodynamic limit. An immediate consequence is that multi-point correlation functions in the steady state decouple into products of one-point functions, allowing us to find the exact expression for the current of each species:

$$
J_{1}=\left(r_{1}-r_{2}\right) \rho_{1}^{o}\left(1-\rho_{1}^{o}\right)\left(1-2 \rho_{2}^{o}\right)
$$

for the $\sigma$-particle current, and a similar expression with 1 and 2 interchanged for the $\tau$-particle current.

Hydrodynamic equations for density fluctuations can be derived using these expressions for the current, on following the procedure of Section 1.1. Further, by taking linear combinations of the fields, one can construct eigenmode fields $h_{ \pm}$which decouple at the linear level. These fields describe wave-like modes travelling with speeds $c_{ \pm}$. The time evolution of the field $h_{+}$is governed by the equation

$$
\frac{\partial h_{+}}{\partial t}=c_{+} \frac{\partial h_{+}}{\partial x}+D \frac{\partial^{2} h_{+}}{\partial x^{2}}+\text { Quadratic Nonlinearities }-K\left(\frac{\partial h_{+}}{\partial x}\right)\left[\left(\frac{\partial h_{+}}{\partial x}\right)^{2}-\left(\frac{\partial h_{-}}{\partial x}\right)^{2}\right]+\eta_{+}(x, t)
$$

and a similar equation holds for $h_{-}$. The quadratic nonlinearities in this equation are of the same form as in Eq. 11. The coefficients of these nonlinear terms, as also the coefficient $K$ of the cubic nonlinearity in Eq. 13, depend on the densities $\rho_{1}^{o}$ and $\rho_{2}^{o}$. The new noise terms $\eta_{ \pm}$are also delta-correlated. While the fields $h_{+}$and $h_{-}$are decoupled at the linear level, they are coupled through the nonlinear terms, so that each mode can influence the dissipation of the other.

Some of the coefficients in Eq. 13 vanish for certain choices of densities $\rho_{1}^{o}$ and $\rho_{2}^{o}$. Then, special symmetries arise in the evolution equations and the dynamical exponents associated with the wave modes may change. Four cases arise.

(a) $R I$ symmetry: Invariance under up-down reflection $(R) h \rightarrow-h$ and under inversion $(I)$ of space $x \rightarrow-x$.

(b) $R \bar{I}$ symmetry: Invariance under $h \rightarrow-h$ and not under $x \rightarrow-x$.

(c) $\bar{R} I$ symmetry: Invariance under $x \rightarrow-x$ and not under $h \rightarrow-h$.

(d) $\bar{R} \bar{I}$ symmetry: Invariance neither under $x \rightarrow-x$, nor under $h \rightarrow-h$.

A term like $\frac{\partial^{2} h}{\partial x^{2}}$ obeys $R I$ symmetry. Terms like $\left(\frac{\partial h}{\partial x}\right)$ and $\left(\frac{\partial h}{\partial x}\right)^{3}$ obey $R \bar{I}$ symmetry. The $\bar{R} I$ symmetry is respected by the term $\left(\frac{\partial h}{\partial x}\right)^{2}$, while a term like $\left(\frac{\partial h}{\partial x}\right)$ added to it breaks that and gives rise to $\bar{R} \bar{I}$ symmetry.

To illustrate the occurrence and effects of different types of symmetries, we consider three special pairs of densities $\left(\rho_{1}^{o}, \rho_{2}^{o}\right)$.

(I) For $\rho_{1}^{o}=\rho_{2}^{o}=\frac{1}{2}$, Eq. 13 and its partner for $h_{-}$reduce to a pair of coupled equations, with linear first and second derivative terms and cubic gradient nonlinearities. Quadratic nonlinearities are absent.

The equations describe two kinematic waves moving with speed $c_{+}=r^{\prime} / 2$ and $c_{-}=-r^{\prime} / 2$ where $r^{\prime}=r_{1}-r_{2}$. The nonlinear couplings imply that each wave influences the evolution of the other. In order to study the dissipation of say the + mode, it is essential to move to the frame which co-moves with it, through the Galilean shift $x \rightarrow x+c_{+} t$, $t \rightarrow t$.

Evidently, in this frame, the - mode has a speed $c_{-}-c_{+}$. The evolution equation is invariant under $h_{+} \rightarrow-h_{+}$, $h_{-} \rightarrow-h_{-}$but not $x \rightarrow-x$, because of the linear $\frac{\partial h_{-}}{\partial x}$ and cubic nonlinear terms. The $R \bar{I}$ symmetry holds in the rest frame of $h_{+}$mode. Similarly the dissipation of the -mode can be studied by going to a frame which co-moves with it. It is easily seen that $R \bar{I}$ symmetry holds in this frame as well.

Each of the + and - modes thus have the same symmetry as a single scalar field evolving through Edwards-Wilkinson dynamics with additional cubic nonlinearities. For the corresponding single-field system, we have $z=2$ (though with multiplicative powers of logarithms) [31], and we might expect the same behaviour here.

(II) For $\rho_{1}^{o}=1 / 2$ and $\rho_{2}^{o} \neq 1 / 2$, on going to either of the frames in which the + mode or the - mode are at rest, we see that the $\bar{R} \bar{I}$ symmetry applies for each of the fields; the $R$ symmetry is broken by by quadratic nonlinear terms, and $I$ is broken because of linear first order and cubic terms. The most relevant terms at the linear fixed point are the quadratic nonlinear terms. Thus we would expect these terms to govern the dissipation and give rise to the KPZ value $z=3 / 2$ [32] for both the modes.

(III) For $\rho_{1}^{o}=\rho_{2}^{o} \neq 1 / 2$, an interesting situation arises. In the co-moving frame of the -mode, the pair of equations have symmetry under $h_{-} \rightarrow-h_{-}$and $h_{+} \rightarrow h_{+}$but not under $x \rightarrow-x$. Thus the $h_{-}$field has $R \bar{I}$ symmetry, while the moving $h_{+}$field has $\bar{R} \bar{I}$ symmetry. The same symmetries hold in the rest frame of the + wave. Based on these 
observations, we expect $z=2$ for the - mode (perhaps with multiplicative logarithmic corrections), and $z=3 / 2$ for the + mode.

Thus based on simple considerations of symmetry, we would expect different types of behaviour in the cases I, II and III. These expectations are borne out by both numerical simulations and analytic calculations. The equal-space unequal-time autocorrelation function $F(t) \sim t^{\beta}$ for the lattice model was investigated numerically for the three cases discussed above. Our Monte Carlo results for $\beta$ are in accord with expectations based on symmetries of the continuum equations. In case I, we find $\beta=1 / 4$ with evidence for multiplicative logarithmic corrections for both modes. In case II, we find $\beta=1 / 3$ for both modes, indicating KPZ behaviour. Finally, in case III, we find that one mode has type I behaviour, while the other one has type II behaviour, as expected on grounds of symmetry.

An analytical treatment of the generalised Langevin equations (13) is straightforward to carry out 23. The renormalised propagators and correlation functions for $h_{+}$and $h_{-}$are calculated in a 1-loop self-consistent scheme. In case III in particular the character of the perturbation theory is controlled by the presence of kinematic waves. In contrast to what happens in [11], their effect cannot be shifted away by Galilean transformations, and we find as expected from the symmetry arguments above that $z_{+}=3 / 2$ and $z_{-}=2$. The calculations for the other cases (I and II) likewise give results consistent with the symmetry arguments above.

\section{CONCLUSION}

We conclude by pointing out some connections to other models and discussing some open questions.

As discussed in Section 2, the strongly phase separated phase of the LR model is closely related to the corresponding phase of the permutation-symmetric ABC model 18,19 especially at the symmetric point of each model where the detailed balance condition is valid. However, an analog of the threshold case of fluctuation-dominated phase ordering which occurs in the LR model has not yet been found in the ABC or related models. In particular, the permutation non-symmetric three-component model of Arndt et al [33] shows a transition from a phase-separated phase to a disordered phase with a large correlation length [34], but the state at the transition point does not seem to show FDPO.

The threshold case of the LR model is tantamount to the problem of hard-core particles sliding down a fluctuating surface. This leads to the question of whether fluctuation-dominated phase ordering survives if the surface in question evolves differently. A study of the problem with KPZ dynamics for the surface shows that FDPO survives [22], though the value of the cusp exponent changes from that in the EW case. On the other hand, with the surface evolving according to Das Sarma-Tamborenea dynamics [35], there is no cusp in $C(y)$ (Eq. 9), and the Porod law is recovered [22]. Another interesting direction to investigate is what happens if the falling particles are noninteracting. Some results in this direction are available from a study of domain-wall dynamics in a model of growth of binary films [36].

The continuum equations of motion in the wave phase are a one-dimensional reduced model of those governing the dynamics of small fluctuations of a flux lattice drifting through a clean type II superconductor [12]. These equations, or more properly the equations implied by (1) for $\partial_{x} h_{1}, \partial_{x} h_{2}$, with some of the couplings set to zero, are Burgers-like equations for magnetohydrodynamics (MHD), as shown in [11]. The dynamics in this kinematic-wave region of the phase diagram is largely governed by KPZ-like critical exponents (in particular $z=3 / 2$ ). On special loci in the space of parameters, however, weak dynamic scaling prevails: if $h_{ \pm}$are the eigenmodes of the linearised versions of the equations, then the complete nonlinear theory on these loci is governed by $z_{+}=3 / 2, z_{-}=2$ for $h_{+}$and $h_{-}$ respectively 23].

Finally, an important open question concerns the behaviour of the LR lattice model in higher dimensions. Recently, it has been shown that a higher-dimensional version of the ABC model continues to show strong phase separation [37. It would be interesting to investigate analogous questions for all phases of the LR model as well. So far, the only result along these lines is the demonstration of product measure along particular loci in the disordered phase in higher dimensions 23].

\section{ACKNOWLEDGEMENT}

The work summarised in this article began in collaboration with Rangan Lahiri whose untimely death in 1998 ended a scientific career of great promise. We dedicate this paper to his memory. 
* Also at Jawaharlal Nehru Institute for Advanced Scientific Research, Bangalore, India.

ansriram@physics.iisc.ernet.in

brbarma@theory.tifr.res.in

c dibyendu@octane.cc.brandeis.edu

d abhik@physics.iisc.ernet.in

[1] B. Schmittmann and R.K.P. Zia, in Phase Transitions and Critical Phenomena, edited by C. Domb and J.L. Lebowitz (Academic Press, London, 1995), Vol. 17.

[2] D. Ertaş and M. Kardar, Phys. Rev. E 48, 1228 (1993).

[3] D. Ertaş and M. Kardar, Phys. Rev. Letts. 69, 929 (1992).

[4] M. Kardar, cond-mat/9704172.

[5] A.-L. Barabási, Phys. Rev. A 46, R2977 (1992).

[6] A.-L. Barabási, Phys. Rev. Letts. 70, 4102 (1993).

[7] R. Lahiri and S. Ramaswamy, Phys. Rev. Lett. 79, 1150 (1997).

[8] J.M. Crowley, J. Fluid Mech. 45, 151 (1971).

[9] J.M. Crowley, Phys. Fluids 19, 1296 (1976).

[10] S. Ramaswamy, Current Science 77, 402 (1999).

[11] S. Yanase, Phys. Plasmas 4, 1010 (1997); J. Fleischer and P.H. Diamond, Phys. Rev. E 58, R2709 (1998); A. Basu, J.K. Bhattacharjee, and S. Ramaswamy, Eur. Phys. Jour. B 9725 (1999)

[12] R. A. Simha and S. Ramaswamy, Phys. Rev. Lett. 83, 3285 (1999).

[13] P. Biswas, A. Majumdar, A. Mehta and J. K. Bhattacharjee, Physica A 248, 379 (1998).

[14] R. Lahiri, M. Barma and S. Ramaswamy, Phys. Rev. E 61, 1648 (2000)

[15] D. Mukamel, cond-mat/0003424.

[16] M. R. Evans, Brazilian Journal of Physics 30, 42 (2000).

[17] H. Hinrichsen, cond-mat/0006212.

[18] M.R. Evans, Y. Kafri, H. M. Koduvely and D. Mukamel, Phys. Rev. Lett. 80, 425 (1998)

[19] M.R. Evans, Y. Kafri, H.M. Koduvely and D. Mukamel, Phys. Rev. E 58, 2764 (1998).

[20] D. Das and M. Barma, Phys. Rev. Lett. 85, 1602 (2000).

[21] M.J. Lighthill and G.B. Whitham, Proc. R. Soc. A 229, 281, 317 (1955).

[22] D. Das, S. N. Majumdar and M. Barma, cond-mat/0102521

[23] D. Das, A. Basu, M. Barma and S. Ramaswamy, cond-mat/0101005

[24] G. M. Schütz. R. Ramaswamy and M. Barma, J. Phys. A: Math. Gen. 29837 (1996).

[25] L. D. Landau and E. M. Lifshitz, Statistical Physics, (Pergamon Press, London, 1969).

[26] M. Barma and S. Ramaswamy, Turkish J. Phys. 244, 235 (2000).

[27] S. F. Edwards and D. R. Wilkinson, Proc. R. Soc. London, Ser A 381, 17 (1982).

[28] A. J. Bray, Adv. Phys. 43, 357 (1994).

[29] G. Korniss, B. Schmittmann and R.K.P. Zia, Europhys. Lett. 45, 431 (1999).

[30] S.N. Majumdar, C. Sire, A.J. Bray, and S.J. Cornell, Phys. Rev. Lett. 77, 2867 (1996).

[31] M. Paczuski, M. Barma, S. N. Majumdar, and T. Hwa, Phys. Rev. Lett. 69, 2735 (1992).

[32] M. Kardar, G. Parisi and Y.C. Zhang, Phys. Rev. Lett. 56, 889 (1986).

[33] P. F. Arndt, T. Heinzel and V. Rittenberg, J. Stat. Phys. 97, 1 (1999).

[34] N. Rajewsky, T. Sasamoto, E.R. Speer, cond-mat/9911322.

[35] S. Das Sarma and P. Tamborenea, Phys. Rev. Lett. 66, 325 (1991).

[36] B. Drossel and M. Kardar, cond-mat/0002032.

[37] Y. Kafri, D. Biron, M.R. Evans, and D. Mukamel, Euro. Phys. J B 16, 669 (2000). 